\title{
Lithocholate Glucuronide Is a Cholestatic Agent
}

David G. Oelberg, Mohan V. Chari, Joanna M. Little, Eugene W. Adcock, and Roger Lester Division of Gastroenterology, Department of Internal Medicine, and Department of Pediatrics, University of Texas Medical School at Houston, Houston, Texas 77225

bstract. Lithocholic acid and its taurine, glycine, and sulfate derivatives are potent cholestatic agents. Lithocholate glucuronide is present in the plasma and urine of patients with cholestatic syndromes, but little is known of its metabolism, excretion, and cholestatic potential. $\left[3 \beta-{ }^{3} \mathrm{H}\right] \mathrm{lithocholate} \quad 3-\mathrm{O}-\beta$-D-glucuronide was synthesized, and chemical and radiochemical purity were established. The aqueous solubility of lithocholate glucuronide was determined and found to be greater than that of lithocholic acid or several of its derivatives. In the range of concentrations examined, calcium ions precipitated lithocholate glucuronide stoichiometrically. The material was administered to rats prepared with an external biliary fistula. When 17-25- $\mu$ g quantities were administered, $89.1 \pm 4.5 \%$ (mean \pm SEM) of the radiolabel was secreted in bile within the first $20 \mathrm{~h}$ after administration, the major fraction being secreted in $<20 \mathrm{~min}$. Four-fifths of the radiolabeled material in bile was the administered unaltered parent compound, while a minor fraction consisted of a more polar derivative(s). We showed that increasing biliary concentrations of more polar derivatives were observed with milligram doses of $\left[{ }^{3} \mathrm{H}\right]$ lithocholate glucuronide, and with time after the administration of these loading doses. Milligram doses of $\left[{ }^{3} \mathrm{H}\right]$ lithocholate glucuronide resulted in partial or complete cholestasis. When induced cholestasis was partial, secretion in bile remained the primary excretory route (82.5-105.6\% recovery in bile), while, when complete cholestasis was induced, wide tissue distribution of ra-

These results were presented in part at the American Association for the Study of Liver Diseases meeting in Chicago, IL, November 1983, and appear in abstract form in Hepatology (Baltimore). 3:857, 1983.

Address correspondence to Dr. Lester.

Received for publication 10 November 1983 and in revised form 6 February 1984.

J. Clin Invest.

(c) The American Society for Clinical Investigation, Inc.

0021-9738/84/06/1507/08 $\$ 1.00$

Volume 73, June 1984, 1507-1514 diolabel was observed. Cholestasis developed rapidly during infusion of $\left[{ }^{3} \mathrm{H}\right]$ lithocholate glucuronide. Bile flow was diminished within 10-20 min of the start of an infusion of $0.05 \mu \mathrm{mol}, 100 \mathrm{~g}^{-1}$ body weight, minute ${ }^{-1}$, administered concomitantly with an equimolar infusion of taurocholate. The results establish that lithocholate glucuronide exerts cholestatic effects comparable to those exerted by unconjugated lithocholic acid.

\section{Introduction}

Lithocholic acid is a secondary bile acid, formed in the colon by the bacterial $7 \alpha$-dehydroxylation of chenodeoxycholic acid (1). Small amounts of lithocholic acid are reabsorbed from the colon, cleared from the portal blood by the liver, and metabolized. Hepatic metabolism results in the formation of taurine and glycine conjugates linked by amide bonds with the steroid carboxylic acid, or in the formation of 3-sulfate esters, or in combined sulfated conjugates (2).

More recently it has been shown that the glucuronide conjugate of lithocholic acid is present in quantity in the serum and urine of patients with cholestatic liver disease (3-6). The conjugate is the 3-O- $\beta$-D-glucuronide of lithocholic acid ${ }^{1,2}$ (LCG) (Fig. 1). The accumulation of LCG in blood in such patients suggests that it may normally be secreted in bile, but that it accumulates in blood when hepatic secretion fails. Surprisingly little, however, has been established about the normal metabolic fate of the substance.

Accordingly, we have prepared radiolabeled LCG and administered it to rats prepared with a biliary fistula. The results show that when microgram quantities of the material are injected intravenously into rats, the material is secreted in bile largely

1. Abbreviations used in this paper: DEAP, diethylaminohydroxypropyl; FAB-MS, fast atom bombardment-mass spectrometry; GC, gas chromatography; LCG, 3-O- $\beta$-D-glucuronide of lithocholic acid; HPLC, highpressure liquid chromatography; Rf, retardation factor; Rt, retention time; $\mathbf{R v}$, retention volume.

2. For the purpose of this paper, the free acid and salt forms of the glucuronide will be considered equivalent and will henceforth be referred to by the abbreviation LCG. 
A<smiles>CC(CCC(=O)O)C1CCC2C3CCC4CC(O)CCC4(C)C3CCC12C</smiles>

Figure 1. Structure of lithocholic acid $(A)$ and lithocholate 3-O- $\beta$-D-glucuronide $(B)$.

intact. When larger quantities are administered, cholestasis results, additional metabolites are formed, and radiolabel accumulates in tissues.

\section{Methods}

Experimental design. $\left[{ }^{3} \mathrm{H}\right] \mathrm{LCG}$ was administered intravenously to rats prepared with an external biliary fistula. The amounts administered varied from microgram to milligram amounts, and the mode of administration was by bolus injection or constant infusion. Radiolabel in bile, urine, plasma, viscera, and carcass was determined. The nature of the radiolabeled material secreted in bile was determined chromatographically, before and after specific enzymatic hydrolysis, and by fast atom bombardment-mass spectrometry (FAB-MS).

Synthesis of lithocholate glucuronide. Methyl lithocholate (Calbiochem-Behring, La Jolla, CA) was oxidized with Jones reagent to the 3-oxo derivative. Subsequent reduction with $\mathrm{NaB}^{3} \mathrm{H}_{4}$ (New England Nuclear, Boston, MA; specific activity, $110 \mathrm{mCi} / \mathrm{mmol}$ ) yielded a mixture

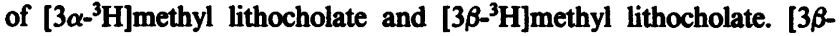
$\left.{ }^{3} \mathrm{H}\right]$ methyl lithocholate, which was the major product (80\%), was separated by preparative thin-layer chromatography (TLC) on silica gel G plates $(20 \times 20 \mathrm{~cm} ; 0.50-\mathrm{mm}$ layer thickness; Supelco, Inc., Bellefonte, PA) using benzene-ethyl acetate (95:5) (multiple development). To prepare the glucuronide conjugate, $\left[3 \beta^{3} \mathbf{H}\right]$ methyl lithocholate was condensed with methyl 2,3,4-tri-O-acetyl-1-bromo-1-deoxy- $\alpha$-D-glucopyranuronate in benzene using silver carbonate as catalyst and molecular sieve $(3 \AA)$ as dessicant (7). The reaction mixture was treated with 0.05 $\mathrm{N}$ sulfuric acid in acetone $(15 \mathrm{~min})$ to decompose the ortho ester side product and was subjected to deprotection in $1 \mathrm{~N} \mathrm{NaOH}$ in $72 \%$ ethanol, room temperature, for $\mathbf{4 8} \mathrm{h}$. The product was acidified with $10 \% \mathrm{HCl}$, passed through an XAD-2 (Aldrich Chemical Co., Milwaukee, WI) column $(1 \times 20 \mathrm{~cm})$ and eluted with $2 \%$ ammonia in methanol. The eluate, containing the ammonium salt of the LCG, was passed over an Amberlyst A-15 (Aldrich Chemical Co.) column $(1 \times 12 \mathrm{~cm})$ with the effluent flowing directly onto a column $(0.75 \times 12 \mathrm{~cm})$ of diethylaminohydroxypropyl (DEAP)-Sephadex LH-20 (prepared as described in [8]). The latter column was washed with $70 \%$ methanol to remove neutral compounds, and the glucuronide was separated from unreacted lithocholic acid by stepwise elutions with acetic acid-ammonia buffer solutions (8). Lithocholic acid was eluted first with $0.1 \mathrm{M}$ acetic acid buffer, pH 3.8, after which the glucuronide was recovered from the column with $0.3 \mathrm{M}$ acetic acid-ammonia buffer, pH 5. Eluate fractions were treated with two drops of $10 \%$ ammonia in methanol and the residue

was subjected to lyophilization to remove all traces of ammonium acetate. The resultant $\left.{ }^{3} \mathrm{H}\right] \mathrm{LCG}$ diammonium salt was $97 \%$ radiochemically pure, as demonstrated by TLC in two solvent systems described below.

The product of the analogous reaction using unlabeled methyl lithocholate and the bromosugar was subjected to column chromatography on silica gel using benzene-ethyl acetate (1:1) as eluent. The protected LCG was eluted immediately after the ortho ester side product and, on recrystallization from ethanol, formed colorless needles, $180-182^{\circ} \mathrm{C}$ melting point. The crystalline material was deprotected as above. The solution was evaporated to dryness and the disodium salt of LCG was recrystallized from $72 \%$ aqueous ethanol, forming crystalline plates that decomposed above $260^{\circ} \mathrm{C}$. FAB-MS spectra were obtained as described elsewhere. The disodium salt of $\mathrm{LCG}$ was characterized by its ${ }^{1} \mathrm{H}$-nuclear magnetic resonance spectrum in $\mathrm{D}_{2} \mathrm{O}$ at room temperature. In addition to the expected signals for the steroid moiety between 0.8 and $2.2 \mathrm{ppm}$, the spectrum exhibited signals for five protons in the region 3.2-4.8 $\mathrm{ppm}$ due to the glucuronide residue. The H-1' signal appeared as a doublet $(\mathrm{J}=8 \mathrm{~Hz})$ centered at $4.6 \mathrm{ppm}$, which is characteristic for a $\beta$-D-glucopyranuronide.

Surgical preparations. $200-600-g$ adult male Sprague-Dawley rats were anesthetized with ether. The right or left femoral triangle was incised and a polyethylene catheter $(0.58 \mathrm{~mm}$ i.d., $0.965 \mathrm{~mm}$ o.d.; ClayAdams, Parsippany, NJ) was inserted in the femoral vein. Thereafter, a constant infusion of $5 \%$ dextrose in $0.2 \%$ sodium chloride solution was maintained at the rate of $15-20 \mathrm{ml}, 100 \mathrm{~g}^{-1}$ body weight, day ${ }^{-1}$ for the duration of the experiment, except when changed by the experimental protocol. The femoral incision was closed, and a midline abdominal incision provided entry to the peritoneal cavity. The common bile duct was isolated and ligated just proximal to the entry of the pancreatic ducts. A polyethylene catheter $(0.28 \mathrm{~mm}$ i.d., $0.61 \mathrm{~mm}$ o.d.) was then inserted in the common bile duct proximal to the ligature. The biliary catheter was externalized through an abdominal stab wound and the abdominal incision was closed. The rat was placed in a Bollman restraining cage and was allowed $3 \mathrm{~h}$ for recovery from the effects of anesthesia and surgery before we proceeded with the experiment. Body temperature was monitored and maintained at $37^{\circ}-38^{\circ} \mathrm{C}$.

Experimental protocol. Three groups of rats were studied (Table I). The first group (rats 1-4) received a bolus intravenous injection of microgram quantities of $\left[{ }^{3} \mathrm{H}\right] \mathrm{LCG}$ dissolved in $2.0 \mathrm{ml}$ normal saline. The second group (rats 5-8) received an injection over 1-2 min of milligram quantities of $\left.{ }^{3} \mathrm{H}\right] \mathrm{LCG}$ dissolved in $4.0-7.0 \mathrm{ml}$ normal saline. The third group (rats 9-12) received successive 1-h infusions of normal saline, normal saline plus sodium taurocholate delivered at $0.05 \mu \mathrm{mol}$, $100 \mathrm{~g}^{-1}$ body weight, minute ${ }^{-1}$, and normal saline, taurocholate (rate of delivery as before), and $\left[{ }^{3} \mathrm{H}\right] \mathrm{LCG}$, the latter delivered for $1 \mathrm{~h}$ each at rates of $0.05,0.10,0.15 \mu \mathrm{mol}, 100 \mathrm{~g}^{-1}$ body weight, minute ${ }^{-1}$. For the sixth 1-h period saline and taurocholate were infused, and, thereafter, until $20 \mathrm{~h}$, the time of sacrifice, rats were infused with $5 \%$ dextrose in $0.2 \%$ saline.

Bile was collected in preweighed tubes and bile volumes were determined gravimetrically. Urine was collected as a single sample for the entire experimental period. Blood was collected at the time of sacrifice from the abdominal aorta, and plasma was separated and frozen. In one rat, blood samples were obtained every $15 \mathrm{~min}$ during the equimolar infusion of taurocholate and $\left[{ }^{3} \mathrm{H}\right] \mathrm{LCG}\left(0.05 \mu \mathrm{mol}, 100 \mathrm{~g}^{-1}\right.$ body weight, minute $\left.{ }^{-1}\right)$. Liver, intestine, and kidneys from all animals were removed and frozen for later analysis. Lung, heart, brain, skin, and muscle were removed and analyzed as noted in Table $I$.

Analytical techniques. For measurement of radiolabel in biological tissues, samples were homogenized in 5-20 ml of distilled water. Aliquots 
of the homogenates were suspended in a mixture of $95 \%$ ethanol-ammonia $(1000: 1 \mathrm{v} / \mathrm{v})$, heated at $75^{\circ}-80^{\circ} \mathrm{C}$ for $30 \mathrm{~min}$, and then centrifuged at $1,000 \mathrm{~g}$ for $10 \mathrm{~min}$. The total weights of skin and muscle mass per rat were calculated as 18.0 and $45.4 \%$ of total body weight, respectively (9). Aliquots of bile, urine, plasma, and ethanol extract of tissue homogenates were analyzed for radiolabel in ACS scintillant (Amersham Corp., Arlington Heights, IL) in a Tracor Mark III, model 6882, liquid scintillation system (Tracor Analytic Inc., Elk Grove Village, IL).

Bile samples were analyzed chromatographically on Whatman LK-5 thin-layer plates (Pierce Chemical Co., Rockford, IL). Isooctaneethyl acetate-glacial acetic acid (50:50:2.5) (solvent system I) and chloroform-methanol-glacial acetic acid-water (65:25:2:4) (solvent system II) were employed for the chromatography of free and conjugated bile acids, respectively. Plates were developed to $19 \mathrm{~cm}$ from the origin and dried. Standards of free and conjugated bile acids (Calbiochem-Behring) were visualized by spraying with $3.5 \%$ phosphomolybdic acid in isopropanol and heating at $110^{\circ} \mathrm{C}$ for $20 \mathrm{~min}$. Samples lanes were divided into $1-\mathrm{cm}$ segments from the origin to the front, and the gel in each segment was transferred to a scintillation vial. The gel was mixed with $1 \mathrm{ml}$ of methanol for $30 \mathrm{~min}$ before counting the mixture in ACS scintillant fluid.

To prepare bile samples for high-pressure liquid chromatography (HPLC) and gas chromatography (GC), aliquots were acidified and extracted with diethyl ether $(80-90 \%$ of the label in bile was recovered in the ether phase). The ether extracts, containing the $\left[{ }^{3} \mathrm{H}\right] \mathrm{LCG}$, were divided in half and dried. One portion was used without further manipulation for HPLC analysis and the second was converted to the methyl ester acetate derivative (10) before $\mathrm{GC}$ analysis.

HPLC analysis was performed with a Waters Associates, Millipore Corp. (Milford, MA) HPLC equipped with a gradient elution system and a model 450 variable wavelength ultraviolet detector set at $196 \mathrm{~nm}$. An LC-18 column (Supelco Inc.) $(5 \mu \mathrm{g}$ particle size; $4.6 \mathrm{~mm}$ ID, $15-\mathrm{cm}$ length) was used with a mobile phase composed of $2 \mathrm{mM}$ phosphate buffer, $\mathrm{pH} 7.0$ (prepared by adjusting $2 \mathrm{mM}$ phosphoric acid to pH 7.0 with $5 \mathrm{~N} \mathrm{NaOH}$ ) and acetonitrile. Bile extracts and steroids were dissolved in the phosphate buffer and 10-20- $\mu$ l aliquots were injected onto the column. Chromatography was carried out at a flow rate of 1 $\mathrm{ml} / \mathrm{min}$ with gradient elution from 0 to $40 \%$ acetonitrile in $2 \mathrm{~min}$ followed by isocratic elution at $40 \%$ acetonitrile for $20 \mathrm{~min}$. Fractions ( $1 \mathrm{ml}$ each) were collected throughout the chromatograph and these were subsequently counted to correlate elution of mass (detected by ultraviolet absorption at $196 \mathrm{~nm}$ ) and label.

The methyl ester acetate derivatives of LCG were analyzed by capillary GC on a H-P 5880-A gas chromatograph (Hewlett-Packard Co., Avondale, PA) equipped with a capillary injector and flame-ionization detector. A short length of DB-1 column (0.25 mm ID, 2.5-m length; J \& W Scientific, Rancho Cordova, CA) was used with nitrogen as carrier gas at $1 \mathrm{psi}$. Samples and standards dissolved in toluene were injected $(0.2-$ $1.0 \mu \mathrm{l})$. Chromatography was performed either isothermally at $300^{\circ} \mathrm{C}$ or under a temperature program from $200-300^{\circ} \mathrm{C}\left(200^{\circ} \mathrm{C}\right.$ for $1 \mathrm{~min}$, then increasing to $300^{\circ}$ at $5^{\circ} \mathrm{C} / \mathrm{min}$ ). Injector and detector temperatures were $290^{\circ}$ and $300^{\circ} \mathrm{C}$, respectively, in both modes of operation.

[ $\left.{ }^{3} \mathrm{H}\right] \mathrm{LCG}$ in bile samples was prepared for FAB-MS analysis by the following chromatographic procedure. Samples were acidified, applied to an Amberlite XAD-2 column, and eluted with $95 \%$ ethanol-0.25\% ammonium hydroxide. The eluate was dried, dissolved in aqueous ethanol, applied to an Amerlyst A-15 column (Aldrich Chemical Co.), eluted with $72 \%$ aqueous ethanol, and allowed to flow directly onto a DEAP Sephadex LH-20 column. This column was eluted with $0.2 \mathrm{M}$ acetate buffer in a pH gradient, 3.9-9.5, and the $\left[{ }^{3} \mathrm{H}\right] \mathrm{LCG}$ was tentatively identified by silica gel TLC developed in ethanol-ethyl acetate-ammonium hydroxide (4.5:4.5:1.0). The $\left.{ }^{3} \mathrm{H}\right] \mathrm{LCG}$ in acetate buffer was converted to the sodium salt with $\mathrm{NaOH}$ and then dried under $\mathrm{N}_{2}$. The final product was then subjected to FAB-MS analysis in a Finnigan MAT $3300 /$ Incos apparatus (Finnigan Corp., Sunnyvale, CA) modified to accept an Ion Tech B1 INF saddle field atomic gun (Ion Tech, Ltd., Peddington, England). The latter was operated with xenon at $0.5 \mathrm{ml} / \mathrm{min}$ at $10 \mathrm{psi}$ and with an accelerating voltage of about $8 \mathrm{keV}$. The analyzer pressure was $\sim 10^{-5}$ torr and the sample was introduced as the disodium salt on a glycerol matrix.

Aliquots of bile were subjected to $\beta$-glucuronidase hydrolysis; the bile was diluted with $0.075 \mathrm{M}$ sodium phosphate (pH 6.8) and mixed with $50 \mathrm{U}$ of Escherichia coli $\beta$-glucuronidase (Sigma Chemical Co., St. Louis, MO) to a final volume of $1.0 \mathrm{ml}$. The mixture was incubated for $12 \mathrm{~h}$ at $37^{\circ} \mathrm{C}$ before acidifying and extracting it with diethyl ether. The ether was evaporated under $\mathrm{N}_{2}$ and the residual crystalline material was redissolved in methanol for TLC. Parallel experiments were performed in which $5 \mathrm{mg}$ of saccharolactone was included in the incubation mixture.

A model 9810 Hewlett-Packard (Hewlett-Packard Co., Loveland, CO) calculator and Hewlett Packard programs for $t$ test were employed to aid data analysis.

Measurement of solubility of $\left[{ }^{3} H\right] L C G$. 5-20 mg of the sodium salts of LCG, lithocholic acid, taurolithocholic acid, glycolithocholic acid, lithocholic acid-3-sulfate, or taurolithocholic acid-3-sulfate were suspended in $0.500 \mathrm{ml}$ of distilled water, $\mathrm{pH} 7.0$, at $25^{\circ} \mathrm{C}$. The suspensions were agitated constantly for $72 \mathrm{~h}$ before the undissolved salts were separated from the solutions by ultracentrifugation at $165,000 \mathrm{~g}$ for $10 \mathrm{~min}$ in a Beckman airfuge ultracentrifuge (Beckman Instruments, Palo Alto, CA). The water in $0.10-\mathrm{ml}$ aliquots of each supernatant was removed by evaporation and the remaining solute was weighed with a Cahn 21 automatic electrobalance (Cahn Instruments, Inc., Cerritos, CA). Measurements were repeated twice for each bile salt.

The influence of calcium on solubility was determined for $\left[{ }^{3} \mathrm{H}\right] \mathrm{LCG}$. 4.0-mM solutions of $\left[{ }^{3} \mathrm{H}\right] \mathrm{LCG}$ or sodium $\left[24-{ }^{14} \mathrm{C}\right]$ taurocholate (New England Nuclear; specific activity, $67 \mu \mathrm{Ci} / \mathrm{mg}$ ) were prepared separately with distilled water, $\mathrm{pH}$ 7.0. Aliquots of the above solutions were mixed with aliquots of $4.0 \mathrm{mM} \mathrm{CaCl}$ and water to provide solutions with 2 $\mathrm{mM}$ initial concentrations of bile salt and a volume of $1.0 \mathrm{ml}$. Initial calcium concentrations were $0,0.1,0.5,1.0$, or $2.0 \mathrm{mM}$. The solutions were equilibrated over $24 \mathrm{~h}$ at $25^{\circ} \mathrm{C}$ before ultracentrifugation. The final concentration of bile salt remaining in solution was determined by measurement of radiolabel.

Finally, aqueous mixtures of $\left[{ }^{3} \mathrm{H}\right] \mathrm{LCG}$ and $\left[{ }^{14} \mathrm{C}\right]$ taurocholate were prepared in molar ratios of $1: 1,1: 4$, and 1:12. These mixtures were then combined in solutions to provide the same initial LCG and calcium concentrations as above. The solutions were equilibrated over $24 \mathrm{~h}$ and the concentrations of $\left[{ }^{3} \mathrm{H}\right] \mathrm{LCG}$ and $\left[{ }^{14} \mathrm{C}\right]$ taurocholate remaining in solution were determined by measurement of radiolabel.

\section{Results}

Recovery of radiolabel after administration of tracer dose of $\left[{ }^{3} \mathrm{H}\right] L C G$. The intravenous administration of $\left[{ }^{3} \mathrm{H}\right] \mathrm{LCG}$ in quantities of $25 \mu \mathrm{g}$ or less ( $<0.02 \mu \mathrm{mol}, 100 \mathrm{~g}^{-1}$ body weight) resulted in rapid biliary secretion of radiolabeled material (Table I). $76.9 \pm 3.6 \%$ (mean $\pm \mathrm{SEM}$ ) of the material was secreted in bile within 30 min of injection (Fig. 2). $89.1 \pm 4.5 \%$ of the material was eventually recovered in bile over the $20 \mathrm{~h}$ before sacrifice. Smaller amounts of radiolabel were recovered in urine and 
Table I. Summary of Experimental Data and Isotope Recoveries for Individual Rats

\begin{tabular}{|c|c|c|c|c|c|c|c|c|c|c|}
\hline \multirow[b]{2}{*}{$\begin{array}{l}\text { Rat } \\
\text { no. }\end{array}$} & \multirow[b]{2}{*}{ Weight } & \multirow[b]{2}{*}{$\begin{array}{l}\text { Administration } \\
\text { protocol }\end{array}$} & \multirow[b]{2}{*}{ Dose } & \multirow[b]{2}{*}{ Cholestasis } & \multicolumn{6}{|c|}{ Isotope recovery (\%) } \\
\hline & & & & & Bile & Urine & Plasma & Viscera ${ }^{*}$ & $\begin{array}{l}\text { Skin, } \\
\text { muscle }\end{array}$ & Totalł \\
\hline & $g$ & & $m g$ & & & & & & & \\
\hline 1 & 269 & Trace dose & 0.017 & No & 89.3 & 0.5 & 0.4 & - & - & 90.2 \\
\hline 2 & 303 & Trace dose & 0.018 & No & 85.1 & 0.5 & 0.3 & - & - & 85.9 \\
\hline 3 & 255 & Trace dose & 0.018 & No & 80.5 & 0.9 & 0.2 & - & - & 81.6 \\
\hline 4 & 448 & Trace dose & 0.025 & No & 101.3 & 0.5 & 0.2 & - & - & 102.0 \\
\hline 5 & 385 & Load by bolus & 3.1 & Partial & 105.6 & 0.7 & 0.0 & 0.2 & - & 106.5 \\
\hline 6 & 354 & Load by bolus & 3.9 & Complete & 41.0 & 0.1 & 2.4 & 5.8 & - & 46.9 \\
\hline 7 & 367 & Load by bolus & 5.4 & Partial & 99.0 & 0.1 & 0.1 & 0.7 & 4.0 & 103.8 \\
\hline 8 & 521 & Load by bolus & 7.7 & Complete & 23.2 & 1.3 & 16.8 & 16.3 & 49.4 & 90.2 \\
\hline 9 & 634 & Load by infusion & 48.6 & Complete & 46.8 & 0.1 & 11.5 & 8.7 & - & 55.6 \\
\hline 10 & 198 & Load by infusion & 21.8 & Partial & 82.5 & 0.1 & 1.6 & 1.9 & - & 84.5 \\
\hline 11 & 449 & Load by infusion & 24.2 & Complete & 52.3 & 1.5 & 2.7 & 9.1 & 42.5 & 105.4 \\
\hline 12 & 479 & Load by infusion & 28.1 & Complete & 46.2 & 1.4 & 8.8 & 10.8 & 48.4 & 106.8 \\
\hline
\end{tabular}

* Viscera include liver, small and large intestines, and kidneys. ‡ Total excludes plasma for rats 5-12 (see text).

plasma $(0.6 \pm 0.1$ and $0.3 \pm 0.1 \%$ of the dose, respectively), while the overall recovery of the administered dose was $89.9 \pm 4.4 \%$.

Analysis of bile from rats receiving a tracer dose of $\left[{ }^{3} \mathrm{H}\right] \mathrm{LCG}$. Bile samples from rats receiving microgram doses of $\left[{ }^{3} \mathrm{H}\right] \mathrm{LCG}$ were subjected to $\beta$-glucuronidase hydrolysis, acidified, and extracted into diethyl ether. $89 \%$ of the radiolabel contained in the sample was ether extractable, and, of this fraction, $80.9 \%$ of the radiolabel, after TLC in solvent system I (designed to separate unconjugated bile acids), migrated with an Rf (retardation factor) of 0.52 , which was identical to that of authentic lithocholic acid. $10.1 \%$ of the radiolabel remained at the origin, and there were no other identifiable bands of radiolabel.

Bile samples were then applied directly to TLC plates and developed in solvent system II (designed to separate bile acid conjugates). $81.0 \%$ of the radiolabel migrated with an $\mathrm{Rf}$ of 0.33 , which was identical to that of authentic LCG. An additional $14.9 \%$ of the radiolabel migrated in a band with an $\mathrm{Rf}$ of 0.13 . No other discrete bands of radiolabel were observed on the chromatogram.

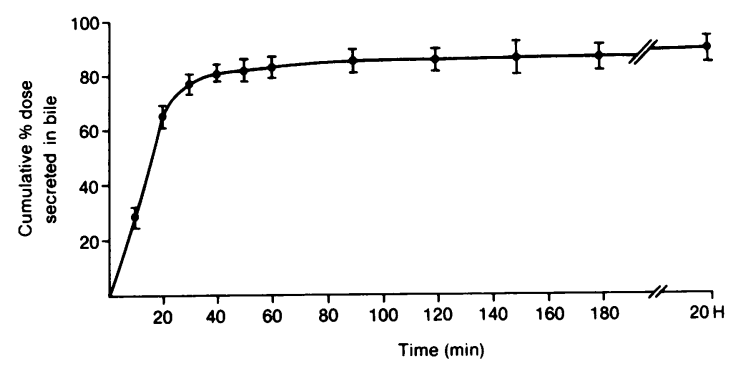

Figure 2. Biliary secretion of $\left[{ }^{3} \mathrm{H}\right]$ lithocholate 3-O- $\beta$-D-glucuronide. Each point represents the mean of four experiments and the brackets give the SEM.
Recovery of radiolabel after administration of a $\left[{ }^{3} \mathrm{H}\right] L C G$ load. Rats 5-8 received 3.1-7.7 mg (1.3-2.6 $\mu \mathrm{mol}, 100 \mathrm{~g}^{-1}$ body weight) of $\left[{ }^{3} \mathrm{H}\right] \mathrm{LCG}$ as a bolus intravenous injection. Bile flow of rats 5 and 7 decreased by 18.5 and $79.6 \%$ of the mean preinjection value within $20 \mathrm{~min}$ of injection. Radiolabel was recovered nearly exclusively in bile (Table I), and total recoveries slightly exceeded $100 \%$. In rats 6 and 8 , bile flow stopped completely within 30 min of injection. 23.2 and $41.0 \%$ of radiolabel was secreted in bile up to the time that flow stopped. Radiolabel was found in plasma, viscera, and, when sought, carcass. Bile became visibly turbid shortly after $\left[{ }^{3} \mathrm{H}\right] \mathrm{LCG}$ administration to rats 6-8; this was attributable to a white crystalline precipitate.

Rats 9-12 received an initial intravenous infusion of taurocholate alone $\left(0.05 \mu \mathrm{mol}, 100 \mathrm{~g}^{-1}\right.$ body weight, minute $\left.{ }^{-1}\right)$ followed by the addition of $\left[{ }^{3} \mathrm{H}\right] \mathrm{LCG}$ at progressively increasing rates of infusion (Fig. 3). Bile flow decreased within $20 \mathrm{~min}$ of the start of the infusion of $\left[{ }^{3} \mathrm{H}\right] \mathrm{LCG}$ at a rate $(0.05 \mu \mathrm{mol}, 100$ $\mathrm{g}^{-1}$ body weight, minute ${ }^{-1}$ ) at which taurocholate and LCG deliveries were equimolar. Plasma LCG concentration rose to $42.5 \mu \mathrm{M}$ within the first $15 \mathrm{~min}$ of the infusion and then doubled over the next $45 \mathrm{~min}$. Bile became turbid in each of the four rats, and complete cessation of flow occurred in three of four. Recoveries in bile and total recoveries of radiolabel from rats receiving an infusion of $\left[{ }^{3} \mathrm{H}\right] \mathrm{LCG}$ were comparable to those obtained in rats receiving a bolus injection (Table I). As would be expected, lesser quantities of radiolabel were secreted in bile when complete cessation of bile flow occurred. Urinary excretion of radiolabel for rats 1-12 was consistently $<2 \%$.

Analysis of bile from rats receiving a load of $\left[{ }^{3} H\right] L C G$. Bile samples from rats 7,9 , and 10 were analyzed systematically by TLC in order to characterize the radiolabeled material in bile; representative results for rat 7 are shown (Fig. 4). Bile samples obtained between 0 and 10, 20 to 30 , and 50 to $60 \mathrm{~min}$ after 

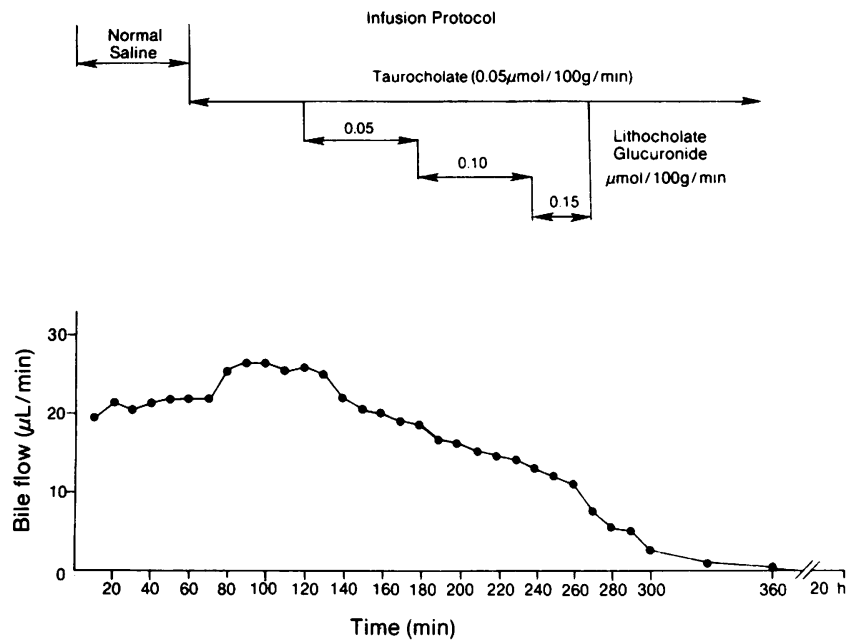

Figure 3. Bile flow rate in rat 9 during infusion of taurocholate and increasing concentrations of lithocholate $3-O-\beta$-D-glucuronide.

injection, when chromatographed in system II, contained 85.9, 43.9 , and $27.1 \%$, respectively, of radiolabel in a discrete band with an $\mathrm{Rf}$ of 0.33 , which was identical to that obtained with authentic LCG. Additional radiolabel was found in a band at an $\mathrm{Rf}$ of 0.13 , and in a separate band at the origin. For bile collected at the three time intervals, percentage of radiolabel in the two bands was $10.1,9.7$, and 35.9 , and $0,43.7$, and 33.0, respectively. Bile samples were then hydrolyzed with $\beta$-glucuronidase, extracted into diethyl ether, and chromatographed on TLC plates in solvent system I. $82.8,66.8$, and $48.1 \%$ of the applied radiolabel migrated with an $\mathrm{Rf}$ of 0.55 , which was identical to that of authentic lithocholic acid, while the bulk of the remainder remained in a single band at the origin. When ether extracts were chromatographed in solvent system II, 8.4, 26.2, and $39.5 \%$ of plated radiolabel migrated with an $\mathrm{Rf}$ of 0.51 , which was identical to that for taurolithocholate. The remainder migrated near the solvent front. 5.4, 33.5, and 39.9\% of radiolabel in bile samples for the three time intervals was not ether extractable. Chromatography in solvent system II of the radiolabel remaining in the aqueous portion of bile samples subjected to $\beta$-glucuronidase hydrolysis and ether extraction established that $90.4,88.3$, and $90.9 \%$ of the radiolabel migrated with an $\mathbf{R f}$ identical to that of taurolithocholic acid. The distribution of radiolabel on chromatograms of bile samples incubated with $\beta$-glucuronidase and saccharolactone was indistinguishable from that of unhydrolyzed samples.

HPLC of bile extracts showed a peak with a retention volume (Rv) of $19.65 \mathrm{ml}$, which corresponded to that of the authentic LCG standard (Rv: $19.68 \mathrm{ml}$ ). When effluent fractions collected during chromatography were analyzed for isotope, there was a single major ( $>90 \%$ of the applied label) radioactive peak with an $\operatorname{Rv}(19.64 \mathrm{ml})$ which, after correction for the lag between detection and collection $(0.25 \mathrm{ml})$, was identical to that of the peak seen in the chromatograph ( $\mathrm{Rv}: 19.65 \mathrm{ml}$ ).
GC analysis of the methyl ester acetate derivatives of bile extracts under isothermal conditions and with temperature programming showed single peaks with retention times $(\mathrm{Rt})$ identical to those of the same derivative of the LCG standard (isothermal: $\mathrm{Rt}$ of $3.58 \mathrm{~min}$ for both extracts and standard; programmed: Rt of $20.00 \mathrm{~min}$ for extracts and 19.99 min for standard).

The white precipitate that appeared in bile after the onset of cholestasis was not present in adequate quantities to isolate for chemical analysis. However, it was readily dissolved in the presence of calcium chelator EDTA or sodium taurocholate, and centrifugation of bile samples containing the precipitate decreased the radiolabel content of the supernatant by $>30 \%$ from the total content.

Further analysis of bile samples from rats receiving $\left[{ }^{3} \mathrm{H}\right] L C G$. Three additional rats received $3-5 \mathrm{mg}\left[{ }^{3} \mathrm{H}\right] \mathrm{LCG}$ each. Bile was collected for $60 \mathrm{~min}$ and the collections were pooled. The samples were subjected to column chromatography on Amberlite XAD-2, Amberlyst A-15, and DEAP Sephadex LH-20 as described above. $60 \%$ of the radiolabel was eluted with an $\mathbf{R v}$ identical to that of authentic LCG (at gradient pH $\sim 8.5$ ), while an additional $23 \%$ was eluted from the column, only under more basic conditions (gradient $\mathrm{pH} \sim 9.0$ ). The larger fraction was converted to the sodium salt, dried under $\mathrm{N}_{2}$, and subjected to FAB-MS. Pseudomolecular ions of the labeled conjugate under FAB conditions were qualitatively identical to those of an authentic sample of lithocholate-3-O- $\beta$-D-glucuronide disodium salt. Ions were observed at $\mathrm{m} / \mathrm{e} 619,597$, and 575 corresponding to $(\mathrm{M}+\mathrm{Na})^{+},(\mathrm{M}+\mathrm{H})^{+}$, and $(\mathrm{M}+\mathrm{H}-\mathrm{Na})^{+}$respectively.

Lithocholate solubility in aqueous solutions. The aqueous solubilities of lithocholic acid and its conjugates were measured (Table II). The glucuronide conjugate was $\sim 20$ times more soluble than the unconjugated parent compound, 50 times more soluble than the amide conjugates, and seven times more soluble than the sulfate conjugate. The solubility of the combined amide-

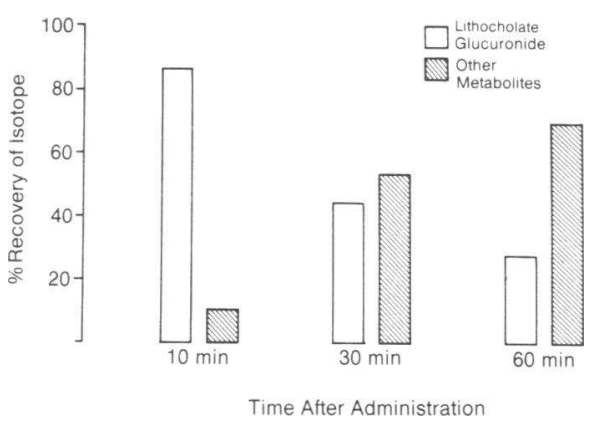

Figure 4. Distribution of isotope in biliary metabolites of lithocholate 3-O- $\beta$-D-glucuronide. Bile samples collected from rat 7 at 10,30 , and $60 \mathrm{~min}$ after administration of $\left[{ }^{3} \mathrm{H}\right]$ lithocholate $3-\mathrm{O}-\beta$-D-glucuronide were analyzed by TLC in solvent system II (see text). Open bars show the percentage of isotope chromatographing with the $\mathbf{R f}$ of standard lithocholate 3-O- $\beta$-D-glucuronide (Rf: 0.33 ), while the hatched bars represent the sum of isotope associated with more polar metabolites (Rf's 0 and 0.13 ). 
Table II. Aqueous Solubility (mM) at $25^{\circ} \mathrm{C}$ of Sodium Salts of Lithocholic Acid and Conjugates

\begin{tabular}{lc}
\hline & Solubility \\
\hline & $m M$ \\
Lithocholic acid & 2.3 \\
Glycolithocholic acid & 1.3 \\
Taurolithocholic acid & 0.8 \\
Lithocholic acid sulfate & 7.5 \\
Taurolithocholic acid sulfate & 40.9 \\
Lithocholic acid glucuronide & 51.7 \\
\end{tabular}

sulfate conjugate, taurolithocholic acid sulfate, was similar to that of LCG.

Table III illustrates the effect of added calcium on LCG solubility. The reported differences between initial and final bile salt concentrations reflect the amount of bile salt precipitated by a given calcium concentration. In the absence of taurocholate, calcium readily precipitated equimolar amounts of LCG. Inclusion of taurocholate decreased LCG precipitation. Calcium had no effect upon $\left[{ }^{14} \mathrm{C}\right]$ taurocholate solubility in the presence or absence of $\left[{ }^{3} \mathrm{H}\right] \mathrm{LCG}$.

\section{Discussion}

It is well established that bile acids are conjugated with taurine and glycine through amide bonds at the steroid side-chain carboxyl group (1). Bile acids also form sulfates, primarily, though not exclusively, through esterification of the 3-hydroxyl group. It is less generally appreciated that glucuronide formation plays a role in bile acid metabolism (3-6). Bile acid glucuronyl transferase is a microsomal enzyme with bile acid specificity inversely proportional to the number of hydroxyl groups on the steroid moiety (4). Enzyme activity is induced by the administration in vivo of phenobarbital and is competitively inhibited by bilirubin (4). Microsomal enzyme activity in vitro is increased by certain detergent substances and might be altered by the detergent properties of bile acids themselves.

It is, therefore, not surprising that bile acid glucuronides are demonstrable in vivo. The amounts found in normal individuals are minute, but greater amounts have been shown to be present in the bile, urine, and plasma of patients with cholestasis $(3,5$, 6). Glucuronides of the full range of conventional human bile acids have been identified, as have atypical bile acid glucuronides. As might be predicted from the specificity of bile acid glucuronyl transferase, glucuronide conjugates of monohydroxylated bile acids are most plentiful, and in some patients may represent $20-30 \%$ of the monohydroxylated bile acid fractions in plasma or urine (5). The relative percentage of bile acid glucuronides increased with phenobarbital treatment of cholestasis (5).

In the present study we have examined the metabolism and disposition of LCG. Bile acid glucuronide metabolism has not been examined in detail previously. The metabolism of LCG is of especial interest since, as noted above, the percentage conversion of monohydroxylated bile acids is considerable, because LCG accumulates in patients with cholestasis, and because of the known toxicity of lithocholate and certain of its other derivatives.

The results show that microgram doses of radiolabeled LCG administered intravenously were rapidly secreted in bile, and that three quarters of the labeled material in bile was unaltered LCG. After hydrolysis with $\beta$-glucuronidase, chromatographic behavior was identical to lithocholate. Before hydrolysis, the radiolabel migrated on TLC identically to authentic LCG's migration, and its FAB-MS spectrum contained fragment peaks identical to those of LCG.

When milligram quantities of $\left[{ }^{3} \mathrm{H}\right] \mathrm{LCG}$ were administered, a portion of the dose was secreted in bile as unaltered LCG; but, as time elapsed after administration, an increasing fraction was secreted as more polar radiolabeled metabolites. The increased polarity was indicated by slower migration in a TLC system that promotes rapid migration of less polar substituents. That at least a major fraction of this more polar material was the taurine conjugate of LCG is strongly suggested by the studies in which the unknown polar conjugate(s) was subjected to hydrolysis with $\beta$-glucuronidase. The chromatographic behavior of the hydrolyzed material was identical to that of authentic taurolithocholic acid, and hydrolysis was completely inhibited by inclusion of the $\beta$-glucuronidase inhibitor, saccharolactone, in the incubation medium. Direct chromatographic comparison of the polar metabolite with taurolithocholate glucuronide was not possible, since an authentic standard of the latter was not available.

Table III. Effect of Calcium on $\left[{ }^{3} \mathrm{H}\right]$ Lithocholate Glucuronide Solubility

\begin{tabular}{|c|c|c|c|c|c|}
\hline & \multirow{3}{*}{$\frac{\begin{array}{l}\text { Initial } \\
\text { [LCG]* }\end{array}}{m M}$} & \multicolumn{4}{|c|}{$\begin{array}{l}\text { Observed differences in } \mathrm{mM} \text { between } \\
\text { the initial and final [ [3 } \mathrm{H} \text { lithocholate } \\
\text { glucuronide concentrations in solution } \\
\text { for each calcium concentration }\end{array}$} \\
\hline & & \multicolumn{4}{|c|}{ Initial $\left[\mathrm{Ca}^{++}\right]$} \\
\hline & & $m M$ & $m M$ & $m M$ & $m M$ \\
\hline & & 0.1 & 0.5 & 1.0 & 2.0 \\
\hline LCG & 1.96 & 0.09 & 0.52 & 1.03 & 1.81 \\
\hline LCG:TC (1:1) & 1.95 & 0.01 & 0.48 & 0.97 & 1.67 \\
\hline LCG:TC (1:4) & 2.00 & 0.03 & 0.46 & 1.00 & 1.76 \\
\hline LCG:TC (1:12) & 1.94 & 0.02 & 0.02 & 0.03 & 0.05 \\
\hline TC $\ddagger$ & 1.98 & 0.10 & 0.12 & 0.07 & 0.10 \\
\hline
\end{tabular}

LCG, lithocholate glucuronide; TC, taurocholate.

* The last value refers to initial [TC].

¥ Values in this line are for decreases in $\left[{ }^{14} \mathrm{C}\right]$ taurocholate concentration (mM). 
To our surprise, LCG proved to be a highly active cholestatic agent. Although rats are less susceptible than humans to lithocholate-induced cholestasis because of alternative metabolic pathways for lithocholate detoxification (11), the plasma concentrations of LCG associated with cholestasis in this model were still within the range of reported human plasma lithocholate concentrations during intrahepatic cholestasis (12). As such, the cholestatic potential of LCG is likely to be of pathophysiologic significance. As shown above, LCG is extremely water soluble in comparison with lithocholate and most of its other metabolites. The activity of lithocholate and its derivatives as cholestatic agents has been thought previously to relate, in part, to their water solubility $(13,14)$. Moreover, it has been suggested that bile acid glucuronide formation, along with sulfation, is an effective mode of detoxification that prevents passage across cell membranes and promotes urinary excretion $(3,5,14-16)$.

Intravenous administration of LCG, however, resulted in partial or complete cholestasis in the rat at doses as low or lower than those at which lithocholate, glycolithocholate, and taurolithocholate produce cholestasis (13-17). Lithocholate sulfate is appreciably less cholestatic than LCG $(14,18,19)$. Most bile acids given in sufficient quantity are capable of producing cholestasis, but the precise mechanism is not fully understood (20). The characteristics of lithocholate that permit it and certain of its derivatives, at low doses, to produce cholestasis have not been adequately explained. It is readily seen, therefore, that no simple explanation is available for the cholestatic properties of another lithocholic acid derivative, LCG. Nevertheless, the solubility characteristics of LCG may be relevant. As shown above, LCG is highly water soluble, but it is precipitated by equimolar concentrations of calcium chloride. LCG is partially or completely maintained in calcium-containing solutions if excess taurocholate is added. In view of the relative concentrations of LCG and other bile acids in bile at the time bile became turbid ( $\sim 2 \mathrm{mM}$ ), LCG precipitation would be anticipated.

It remains to be seen whether LCG precipitation per se initiates cholestasis. After a sufficient dose, bile plugs capable of obstructing the polyethylene catheter are present in bile that are most likely composed of calcium salts of LCG. A similar deposition of LCG might obstruct flow at the ductal, ductular, and/or canalicular level. It has also been suggested that lithocholate and its derivatives produce cholestasis by changing the physical and chemical properties of canalicular membrane, or by altering the vesicular transport across the liver cell of substances destined to enter bile $(12,13,17,21)$. Alternatively, it is tempting to speculate that lithocholate derivatives and, specifically, LCG may bind intracellular calcium and/or change entry of calcium into hepatocytes, thereby altering intracellular calcium concentrations. Were this to occur, changes in a broad spectrum of cellular functions might be anticipated in view of the role of intracellular calcium as a modulator of basic cellular functions (22). Systematic study of lithocholate and its derivatives might offer an interesting probe with which to dissect the pathogenesis of cholestasis and hepatocellular toxicity.

Finally, the role of the increased concentrations of LCG in the plasma of patients with cholestasis should be reconsidered. Lithocholate and other monohydroxylated bile acids may accumulate disproportionately in cholestasis because of increased activity of alternate bile acid biosynthetic pathways (23). Biosynthetic pathways for the glucuronidation of monohydroxylated bile acids may also be stimulated (5). The present results suggest that glucuronidation of lithocholate does not diminish its capacity for producing cholestasis, and in this sense does not appear to be an effective mechanism for detoxification of lithocholate. The accumulation of LCG may be both an effect of cholestasis and a contributory cause for its maintenance.

\section{Acknowledgments}

The authors wish to thank Yvonne Denkins for her technical assistance and Drs. Timothy Tewson and Marc Berridge, Division of Cardiology, for providing the Waters HPLC system and much assistance in establishing the HPLC system used.

This work was supported in part by grants from the National Institute of Child Health and Human Development, Grant No. HD14198, and the March of Dimes Birth Defects Foundation, Grant No. 6-305.

\section{References}

1. Danielsson, H. 1973. Mechanisms of bile acid biosynthesis. In The Bile Acids. Vol. 2. Physiology and Metabolism. P. P. Nair and D. Kritchevsky, editors. Plenum Press, New York. 1-32.

2. Cowen, A. E., M. G. Korman, A. F. Hofmann, O. W. Cass, and S. B. Coffin. 1975. Metabolism of lithocholate in healthy man. I. Biotransformation and biliary excretion of intravenously administered lithocholate, lithocholyglycine, and their sulfates. Gastroenterology. 69:67-76.

3. Frohling, W., and A. Stiehl. 1976. Bile salt glucuronides: identification and quantitative analysis in the urine of patients with cholestasis. Eur. J. Clin. Invest. 6:67-74.

4. Dutton, G. J. 1980. Glucuronidation of drugs and other compounds. CRC Press Inc., Boca Roton, FL.

5. Stiehl, A., M. Becker, P. Czygan, W. Frohling, B. Kommerell, H. W. Rotthauwe, and M. Senn. 1980. Bile acids and their sulphated and glucuronidated derivatives in bile, plasma, and urine of children with intrahepatic cholestasis: effects of phenobarbital treatment. Eur. J. Clin. Invest. 10:307-316.

6. Stiehl, A., R. Raedsch, G. Rudolph, P. Czygan, and S. Walker. 1982. Analysis of bile acid glucuronides in urine: group separation on a lipophilic anion exchanger. Clin. Chim. Acta. 123:275-285.

7. Back, P., and D. V. Bowen. 1976. Bile acid glucuronides. III. Chemical synthesis and characterization of glucuronic acid coupled mono-, di-, and trihydroxy bile acids. Hoppe-Seyler's Z. Physiol. Chem. 357:219-224.

8. Almé, B., A. Bremmelgaard, J. Sjovall, and P. Thomassen. 1977. Analysis of metabolic profiles of bile acids in urine using a lipophilic anion exchanger and computerized gas-liquid chromatography-mass spectrometry. J. Lipid Res. 18:339-362.

9. Donaldson, H. H. The Rat. 1924. Wistar Institute of Anatomy and Biology, Philadelphia. 184.

10. Pyrek, J. St., R. Lester, E. W. Adcock, and A. T. Sanghvi. 1983. Constituents of human meconium. Pt. 1. Identification of 3-hydroxyetianic acids. J. Ster. Biochem. 18:341-351. 
11. Carey, J. B. 1973. Bile salt metabolism in man. In The Bile Acids. Vol. 2. Physiology and Metabolism. P. P. Nair and D. Kritchevsky, editors. Plenum Press, New York. 55-82.

12. Balistreri, W. F., M. H. Leslie, and R. A. Cooper. 1981. Increased cholesterol and decreased fluidity of red cell membranes (spur cell anemia) in progressive intrahepatic cholestasis. Pediatrics. 67:461-466.

13. Javitt, N. B., and S. Emerman. 1968. Effect of sodium taurolithocholate on bile flow and bile acid excretion. J. Clin. Invest. 47:10021014.

14. Yousef, I. M., B. Tuchweber, R. J. Vonk, D. Masse, M. Audet, and C. C. Roy. 1981. Lithocholate cholestasis-sulfated glycolithocholateinduced intrahepatic cholestasis in rats Gastroenterology. 80:233-241.

15. Breemmelgaard, A., and B. Almé. 1980. Analysis of plasma bile acid profiles in patients with liver diseases associated with cholestasis. Scand. J. Gastroenterol. 15:593-600.

16. Balistreri, W. F., F. J. Suchy, M. K. Farrell, and J. E. Heubi. 1981. Pathologic versus physiologic cholestasis: elevated serum concentrations of a secondary bile acid in the presence of hepatobiliary disease. J. Pediatr. 98:399-402.

17. Kakis, G., and I. M. Yousef. 1978. Pathogenesis of lithocholateand taurolithocholate-induced intrahepatic cholestasis in rats. Gastroenterology. 75:595-607.
18. Roy, C. C., I. M. Yousef, N. P. Dorvil, L. A. Fournier, A. Perea, and B. Tuchweber. 1983. The pattern of bile acid conjugation: a critical determinant of the cholestatic potential of sulfolithocholate. Pediatr. Res. 17:199A.

19. Marks, J. W., S. O. Sue, B. J. Pearlman, G. G. Bonorris, P. Varady, J. M. Lachin, and L. J. Schoenfield. 1981. Sulfation of lithocholate as a possible modifier of chenodeoxycholic acid-induced elevations of serum transaminase in patients with gallstones. J. Clin. Invest. 68:1190-1196.

20. Drew, R., and B. G. Priestly. 1979. Choleretic and cholestatic effects of infused bile salts in the rat. Experientia. 35:809-811.

21. Kakis, G., M. J. Phillips, and I. M. Yousef. 1980. The respective roles of membrane cholesterol and of sodium-potassium adenosine triphosphatase in the pathogenesis of lithocholate-induced cholestasis. Lab. Invest. 43:73-81.

22. Rasmussen, H., and D. B. P. Goodman. 1977. Relationships between calcium and cyclic nucleotides in cell activation. Physiol. Rev. 57:421-509.

23. Mitropoulos, K. A., and N. B. Myant. 1967. The formation of lithocholic acid, chenodeoxycholic acid, and alpha- and beta-muricholic acid from cholesterol incubated with rat liver microsomes. Biochem. $J$. 103:472-479. 